\title{
Color Histograms Adapted to Query-Target Images for Object Recognition across Illumination Changes
}

\author{
Damien Muselet \\ Laboratoire LAGIS, UMR CNRS 8146, Université des Sciences et Technologies de Lille, Cité Scientifique, Bâtiment P2, \\ 59655 Villeneuve d'Ascq, France \\ Email:dm@i3d.univ-lille1.fr

\section{Ludovic Macaire} \\ Laboratoire LAGIS, UMR CNRS 8146, Université des Sciences et Technologies de Lille, Cité Scientifique, Bâtiment P2, \\ 59655 Villeneuve d'Ascq, France \\ Email: ludovic.macaire@univ-lille1.fr
}

\section{Jack-Gérard Postaire}

Laboratoire LAGIS, UMR CNRS 8146, Université des Sciences et Technologies de Lille, Cité Scientifique, Bâtiment P2, 59655 Villeneuve d'Ascq, France

Email: jack-gerard.postaire@univ-lille1.fr

Received 14 January 2004; Revised 24 November 2004

\begin{abstract}
Most object recognition schemes fail in case of illumination changes between the color image acquisitions. One of the most widely used solutions to cope with this problem is to compare the images by means of the intersection between invariant color histograms. The main originality of our approach is to cope with the problem of illumination changes by analyzing each pair of query and target images constructed during the retrieval, instead of considering each image of the database independently from each other. In this paper, we propose a new approach which determines color histograms adapted to each pair of images. These adapted color histograms are obtained so that their intersection is higher when the two images are similar than when they are different. The adapted color histograms processing is based on an original model of illumination changes based on rank measures of the pixels within the color component images.
\end{abstract}

Keywords and phrases: color, object recognition, illumination, adapted color histograms, rank measures.

\section{INTRODUCTION}

\subsection{Object recognition by color histogram analysis}

Object searching in a database of color images, which is aparticular problem of color image retrieval, is identical to appearance-based object recognition. In this framework, the recognition problem can be stated in terms of finding among all the target images of a database, those which contain the same object as that represented by the query image. Each of these images contains one single object placed on a uniform background.

In this context, the image indexing scheme consists in extracting robust and efficient characteristic indices from the target and query images. These indices are typically derived from the shape [1], the texture [2], or the color properties [3] of the objects. Object recognition is performed by means of a matching scheme which compares the indices of the query image with those of the target images. The matching scheme is based on a similarity measure between these indices. The target images are ranked with respect to their similarity measures with the query image, in order to determine those which contain the same object as that represented by the query image.

One of the most widely used image indices based on the color distribution is the color histogram [4]. The color histogram $\mathbf{H}[\mathbf{I}]$ of an image $\mathbf{I}$ is composed of bins $\mathbf{H}[\mathbf{I}](\mathbf{c})$ associated with color vectors $\mathbf{c}$ whose coordinates are the levels of the three color components, namely the red $\left(c^{R}\right)$, the green $\left(c^{G}\right)$, and the blue $\left(c^{B}\right)$. Each bin indicates the number of pixels which represent the object in the image and which are characterized by this particular color.

Several similarity measures based on the color histograms have been proposed [5]. For object recognition, a widely used similarity measure between the query image $\mathbf{I}_{\text {que }}$ and one of the target images $\mathbf{I}_{\mathrm{tar}}$ is derived from the intersection Inter $\left(\mathbf{H}\left[\mathbf{I}_{\text {que }}\right], \mathbf{H}\left[\mathbf{I}_{\mathrm{tar}}\right]\right)$ between the color histograms 


$$
\begin{aligned}
& \mathbf{H}\left[\mathbf{I}_{\text {que }}\right] \text { and } \mathbf{H}\left[\mathbf{I}_{\text {tar }}\right][4]: \\
& \operatorname{Inter}\left(\mathbf{H}\left[\mathbf{I}_{\text {que }}\right], \mathbf{H}\left[\mathbf{I}_{\text {tar }}\right]\right) \\
& \quad=\sum_{\mathbf{c}} \min \left(\frac{\mathbf{H}\left[\mathbf{I}_{\text {que }}\right](\mathbf{c})}{\sum_{\mathbf{c}^{\prime}} \mathbf{H}\left[\mathbf{I}_{\text {que }}\right]\left(\mathbf{c}^{\prime}\right)}, \frac{\mathbf{H}\left[\mathbf{I}_{\text {tar }}\right](\mathbf{c})}{\sum_{\mathbf{c}^{\prime}} \mathbf{H}\left[\mathbf{I}_{\text {tar }}\right]\left(\mathbf{c}^{\prime}\right)}\right) .
\end{aligned}
$$

As $\sum_{\mathbf{c}^{\prime}} \mathbf{H}[\mathbf{I}]\left(\mathbf{c}^{\prime}\right)$ represents the number of pixels which represent the object, the intersection requires a segmentation step in order to detect these pixels. When the value of the intersection is close to 1 , the two images have similar colors in similar proportions. Although that does not necessarily mean that the images contain the same objects, we assume so. On the other hand, an intersection close to 0 means that the objects are significantly different.

This measure adds up the minima between the bins associated with the same color vectors. So, when two images contain the same object, this measure is close to 1 only if most of the color vectors of pixels, on which the same elementary surfaces in the two compared images are projected, are equal.

\subsection{Color images across illumination changes}

In this paper, we specifically address the problem of recognizing objects when they are subject to different illumination during the image acquisitions. We consider that the differences result only from temperature and intensity variations. The images acquired under different illumination contain one single object that can be translated or rotated in a plane perpendicular to the optical axis of the camera (see Figure 1). The acquisition parameters of the camera are not modified.

During the retrieval process, two different cases may occur (see Figure 1):

(i) the query image $\mathbf{I}_{\text {que }}$ and the target image $\mathbf{I}_{\text {tar }}$ contain the same object observed with the same viewing conditions but subject to different illumination. They constitute a pair of similar images,

(ii) the images $\mathbf{I}_{\text {que }}$ and $\mathbf{I}_{\text {tar }}$ contain different objects. They constitute a pair of different images.

The color vector of a pixel $P$, denoted $\mathbf{c}(P)=$ $\left[c^{R}(P), c^{G}(P), c^{B}(P)\right]^{T}$, where $c^{k}(P)$ represents the level of the color component $k, k=R, G, B$, is not only a measure of the reflectance properties of the elementary surface of the object projected onto the pixel $P$. The color vector is also a function of both the camera and the illumination [6].

We consider the image of Figure 1a as the query image while one of the images of Figures $1 \mathrm{~b}$ and $1 \mathrm{c}$ is the target image. The color vectors of the pixels which represent the same elementary surfaces of the object in the query and target images are significantly different, whereas these images are similar. That explains why object recognition based on the intersection between color histograms fails in case of illumination changes [7].

\subsection{Invariant color histograms}

Therefore, many authors propose to characterize the images by histograms which are invariant to illumination changes. These histograms are either the histograms of invariant color

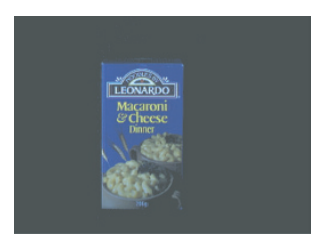

(a)

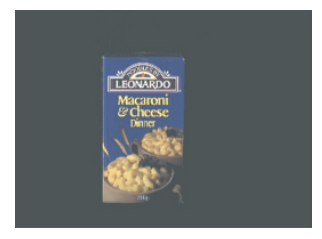

(c)

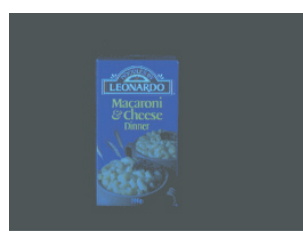

(b)

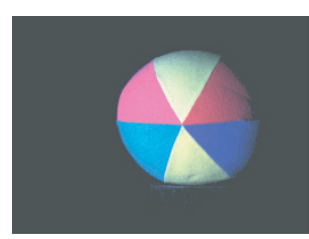

(d)
Figure 1: As the images (a), (b), and (c) contain the same object subject to different illumination during the acquisitions, they are similar. As the image (d) contains another object, the pairs of images $((\mathrm{a}),(\mathrm{d})),((\mathrm{b}),(\mathrm{d}))$, and $((\mathrm{c}),(\mathrm{d}))$ constitute pairs of different images.

images which are derived from the color images, or are obtained by means of transformations of the color histograms of the images.

In the first case, the main image transformation approach consists in correcting the color vectors so that they represent the surface reflectance properties of the object contained in each image (color constancy). The main problem of this approach is the estimation of the illumination when the available information is only the image of the object acquired under an unknown illumination $[8,9]$. Hence, the color constancy approach is not adapted to object recognition when there is no prior knowledge about the acquisition conditions [10].

In the second case, the invariant color histograms of the images are obtained by means of transformations of the color histograms of the images. These transformations are based either on assumptions about color properties or on the analysis of the color distribution. When the transformations are based on assumptions about color properties, they do not depend on the considered image. They are obtained by the color angles analysis [11] or by the "c1c2c3" space which is only invariant for white illumination variations [12].

The greyworld normalization which divides the color component levels by their averages in the image [13], the moments based on the rotation of the color distribution [14], and the principal component analysis of the color distribution [15] yield functions obtained by the analysis of the color distribution with which the color histograms are transformed into invariant color histograms.

The determination of most of these invariant color histograms is based on illumination change models, which describe the variations of color vectors caused by any illumination change. As these models use such restrictive assumptions about the camera and the illumination, they partially take into account the effects of illumination changes on the 
color vectors. That is the reason why object recognition based on the intersection between these invariant color histograms generally performs poorly $[7,16]$.

\subsection{Pair of adapted color histograms}

The main originality of our approach is to cope with the problem of illumination changes by analyzing each pair of query and target images $\left(\mathbf{I}_{\text {que }}, \mathbf{I}_{\text {tar }}\right)$ constructed during the retrieval, instead of considering each image of the database independently from each other.

To be more specific, we propose to analyze each pair of query and target color histograms to compare the query and target images. For this purpose, the pair of color histograms is transformed into a pair of adapted color histograms associated with these two images, so that the intersection between these adapted color histograms is higher when the two images are similar than when they are different. In this case, this intersection allows to discriminate the case when the two compared images are similar from the case when they are different.

The function used for transforming the pair of color histograms into the pair of adapted color histograms takes into account rank measures of the pixels. The image $\mathbf{I}$ is divided into three color component images $I^{k}, k=R, G, B$, where each pixel $P$ is characterized by one single color component level $c^{k}(P)$. Within each color component image, the pixels are sorted in increasing order of level. With the pixels, we associate rank measures which are assumed to be coarsely preserved in case of illumination changes.

\subsection{Paper overview}

This assumption about rank measures is described in Section 2. In Section 3, a new assumption is introduced for defining the function which determines the pair of adapted color histograms from the pair of query and target color histograms. In Section 4, the effectiveness of our object recognition scheme is assessed with a public database of images which contain objects subject to different illumination. In order to demonstrate the improvement of our proposed method, we compare the object recognition results obtained with classical invariant color histograms and those obtained with the adapted color histograms.

\section{RANK MEASURES PROPERTIES}

Most illumination change models describe the modifications of the color vectors of pixels caused by the illumination changes due to linear transformations of the color vectors [17]. This section presents two illumination change models which describe these modifications due to nonlinear transformations. They analyze the rank measures of the pixels within the color component images. Derived from his model, Finlayson assumes that the rank measures of the pixels within the color component images are strictly preserved across the illumination changes [18]. Our proposed assumption, called hereafter the closest rank measures assumption, takes into account the possible modifications of rank measures which may occur when the illumination changes.

\subsection{Rank measures preservation}

\subsubsection{Illumination changes model}

We consider similar query and target images $\left(\mathbf{I}_{\text {que }}, \mathbf{I}_{\mathrm{tar}}\right)$. We examine each of the three pairs of query and target color component images, denoted $\left(I_{\text {que }}^{k}, I_{\text {tar }}^{k}\right), k=R, G, B$, that can be extracted from the pair $\left(\mathbf{I}_{\text {que }}, \mathbf{I}_{\text {tar }}\right)$. We consider two pixels $P_{\text {que }}$ and $P_{\text {tar }}$, called hereafter corresponding pixels, which respectively, belong to the query and target images and on which the same elementary surface of the object contained in these two similar images is projected.

Finlayson [18] stipulates that the level $c^{k}\left(P_{\mathrm{tar}}\right), k=$ $R, G, B$, of the pixel $P_{\text {tar }}$ is deduced from the level $c^{k}\left(P_{\text {que }}\right)$ of its corresponding pixel $P_{\text {que }}$ by means of a monotonic increasing function $f_{\text {que,tar }}^{k}$, which is specific to the pair of color component images $\left(I_{\text {que }}^{k}, I_{\text {tar }}^{k}\right)$ :

$$
c^{k}\left(P_{\text {tar }}\right)=f_{\text {que,tar }}^{k}\left(c^{k}\left(P_{\text {que }}\right)\right), \quad k=R, G, B .
$$

These three monotonic increasing functions $f_{\text {que,tar }}^{k}$ are not a priori linear transformations.

We now consider two different elementary surfaces of the object which are projected onto the pixels $P_{\text {que }}$ and $Q_{\text {que }}$ in the query image and onto the corresponding pixels $P_{\mathrm{tar}}$ and $Q_{\text {tar }}$ in the target image, respectively. If $c^{k}\left(P_{\text {que }}\right)$ is higher than $c^{k}\left(Q_{\text {que }}\right), k=R, G, B$, we deduce from (2) that $c^{k}\left(P_{\text {tar }}\right)$ is higher than $c^{k}\left(Q_{\text {tar }}\right)$ :

$$
\begin{aligned}
& c^{k}\left(P_{\text {que }}\right)>c^{k}\left(Q_{\text {que }}\right) \\
& \Longrightarrow f_{\text {que tar }}^{k}\left(c^{k}\left(P_{\text {que }}\right)\right)>f_{\text {que }, \text { tar }}^{k}\left(c^{k}\left(Q_{\text {que }}\right)\right) \\
& \Longrightarrow c^{k}\left(P_{\text {tar }}\right)>c^{k}\left(Q_{\text {tar }}\right) .
\end{aligned}
$$

\subsubsection{Rank measures}

Within each color component image $I^{k}$, we sort the pixels $P$ in increasing order of level. With each pixel, we associate a normalized rank measure that is close to 0 for the first ordered pixels and equal to 1 for the last pixels. The rank measure $R^{k}[\mathbf{I}](P)$ of the pixel $P$ within the color component image $I^{k}$ is expressed as

$$
R^{k}[\mathbf{I}](P)=\frac{\sum_{l=0}^{c^{k}(P)} H^{k}[\mathbf{I}](l)}{\sum_{l=0}^{L-1} H^{k}[\mathbf{I}](l)}, \quad k=R, G, B,
$$

where $L$ is the number of levels used to quantize the color components ( $L$ is generally set to 256 ), and $H^{k}[\mathbf{I}](l)$ is the number of pixels characterized by the level $l$ in $I^{k}$. Note that this rank measure can be interpreted as the normalized cumulative histogram.

\subsubsection{Rank measures preservation assumption}

From (3), Finlayson [18] assumes that the rank measures of the corresponding pixels $P_{\text {que }}$ and $P_{\text {tar }}$ within each pair of color component images $\left(I_{\text {que }}^{k}, I_{\text {tar }}^{k}\right)$ are equal:

$$
R^{k}\left[\mathbf{I}_{\text {que }}\right]\left(P_{\text {que }}\right)=R^{k}\left[\mathbf{I}_{\text {tar }}\right]\left(P_{\text {tar }}\right), \quad k=R, G, B .
$$


TABle 1: Percentages of pixels with the same spatial coordinates whose rank measures within the green target and query images of Figure 1 are equal $(L=256)$.

\begin{tabular}{l|ccc}
\hline Target image & Query image \\
& Figure 1a & Figure 1b & Figure 1c \\
\hline Figure 1a & $X$ & 7 & 9 \\
Figure 1b & 7 & $X$ & 3 \\
Figure 1c & 9 & 3 & $X$ \\
\hline
\end{tabular}

So, the rank measures of the pixels within each pair of color component images are assumed to be invariant to any illumination change between the acquisitions of similar images.

The histogram of the rank measures of the pixels within each color component image $I^{k}$ is obtained by the wellknown equalization of the 1D histogram $H^{k}[\mathbf{I}]$ (see (4)). Finlayson determines the invariant color histogram by independently equalizing the three $1 \mathrm{D}$ histograms $H^{k}[\mathbf{I}], k=R, G, B$ [18].

\subsubsection{Validity on similar color images}

We propose to verify the above rank measures preservation assumption with the similar color images of Figure 1, where the pixels with the same spatial coordinates in these three images are corresponding pixels. Equation (5) is verified only if the rank measures of the corresponding pixels within the green images are equal. As shown in Table 1, the rank measures preservation is only verified for a few pixels. The bad results shown in Table 1 may be also explained by the acquisition noise. Indeed, even if the same image is captured under the same illumination, there will be shifts in rank measures resulting from the noise. Furthermore, the rank measures preservation assumption does not take into account possible rank modifications due to illumination changes.

\subsection{Closest rank measures}

\subsubsection{Extended Finlayson's model}

We propose to extend the Finlayson model which expresses the relationship between the color component levels of two corresponding pixels in two similar color images. Our proposed model of illumination changes stipulates that the level $c^{k}\left(P_{\mathrm{tar}}\right)$ of the pixel $P_{\mathrm{tar}}$ is expressed from the level $c^{k}\left(P_{\text {que }}\right)$ of its corresponding pixel $P_{\text {que }}$ by

$$
c^{k}\left(P_{\text {tar }}\right)=f_{\text {que }, \text { tar }}^{k}\left(c^{k}\left(P_{\text {que }}\right)\right)+\rho^{k}\left(P_{\text {tar }}\right), \quad k=R, G, B .
$$

When the two images are similar, the bias function $\rho^{k}$ represents the possible modifications of the rank measures of corresponding pixels within the two color component images. These possible rank measures modifications are the consequences of illumination changes between the images.

\subsubsection{Closest rank measures assumption}

From this model, we cannot directly deduce any relation between the rank measures of corresponding pixels. However,
TABle 2: Percentages of pixels with the same spatial coordinates whose rank measures within the green target and query images of Figure 1 are the closest $(L=256)$.

\begin{tabular}{l|ccc}
\hline Target image & \multicolumn{3}{|c}{$\begin{array}{c}\text { Query image } \\
\text { Figure 1b }\end{array}$} \\
\hline Figure 1a & $X$ & 70 & Figure 1c \\
Figure 1b & 70 & $X$ & 33 \\
Figure 1c & 53 & 33 & $X$ \\
\hline
\end{tabular}

we propose a new assumption called hereafter the closest rank measures assumption, which stipulates that each pair of corresponding pixels $\left(P_{\text {que }}, P_{\operatorname{tar}}\right)$ respects the two following properties:

(i) the rank measure of $P_{\text {que }}$ within the color component image $I_{\text {que }}^{k}$ is among all the rank measures of the pixels within $I_{\text {que }}^{k}$, the closest to the rank measure of $P_{\text {tar }}$ within $I_{\text {tar }}^{k}$ :

$$
\begin{aligned}
& \left\|R^{k}\left[\mathbf{I}_{\mathrm{tar}}\right]\left(P_{\mathrm{tar}}\right)-R^{k}\left[\mathbf{I}_{\text {que }}\right]\left(P_{\text {que }}\right)\right\| \\
& =\min _{P \in I_{\text {que }}^{k}}\left\|R^{k}\left[\mathbf{I}_{\mathrm{tar}}\right]\left(P_{\mathrm{tar}}\right)-R^{k}\left[\mathbf{I}_{\text {que }}\right](P)\right\|,
\end{aligned}
$$

(ii) the rank measure of $P_{\text {tar }}$ within the color component image $I_{\mathrm{tar}}^{k}$ is among all the rank measures of the pixels within $I_{\text {tar }}^{k}$, the closest to the rank measure of $P_{\text {que }}$ within $I_{\text {que }}^{k}$ :

$$
\begin{aligned}
& \left\|R^{k}\left[\mathbf{I}_{\text {tar }}\right]\left(P_{\text {tar }}\right)-R^{k}\left[\mathbf{I}_{\text {que }}\right]\left(P_{\text {que }}\right)\right\| \\
& =\min _{P \in I_{\text {tar }}^{k}}\left\|R^{k}\left[\mathbf{I}_{\text {que }}\right]\left(P_{\text {que }}\right)-R^{k}\left[\mathbf{I}_{\text {tar }}\right](P)\right\| .
\end{aligned}
$$

As the rank measures of pixels within the color component images are not always equal, the differences expressed by (7) and (8) are not always equal to 0 . For the sake of simplicity, the rank measures of pairs of pixels which respect to (7) and (8) are referred hereafter as the closest rank measures within the pair of color component images $\left(I_{\text {que }}^{k}, I_{\text {tar }}^{k}\right)$.

This closest rank measures assumption is very original because (7) and (8) do not only take into account the rank measures of two corresponding pixels, but also the rank measures of all the pixels within the query and target color component images for determining the closest rank measures.

\subsubsection{Validity with similar color images}

As for the rank measures preservation assumption, we verify our closest rank measures assumption with the similar color images of Figure 1 (see Table 2). By comparing Tables 1 and 2, the percentages of pixels whose rank measures are the closest are significantly higher than the percentages of pixels with equal rank measures. These results show that our closest rank measures assumption is respected in a higher number of cases than the rank measures preservation assumption. 


\section{COLOR HISTOGRAMS ADAPTED TO QUERY-TARGET IMAGE PAIRS}

For comparing the query and one of the target images, we propose to adapt the query and target color histograms instead of independently determining invariant color histograms of the two images. The intersection between these two adapted color histograms has to be higher when the compared images are similar than when they are different. First, we define the function $\mathbf{T}_{\text {que,tar }}$ which transforms the pair of color histograms $\left(\mathbf{H}\left[\mathbf{I}_{\text {que }}\right], \mathbf{H}\left[\mathbf{I}_{\text {tar }}\right]\right)$ into the pair of adapted color histograms $\left(\mathbf{H}_{\text {que,tar }}\left[\mathbf{I}_{\text {que }}\right], \mathbf{H}_{\text {que,tar }}\left[\mathbf{I}_{\text {tar }}\right]\right)$. Secondly, the pairs of the look-up tables which are associated with the function $\mathbf{T}_{\text {que,tar }}$, are detailed. Finally, we present the scheme which determines these look-up tables.

\subsection{Pair of adapted color histograms}

The pair of adapted color histograms is determined thanks to the analyses of the projections of the pair of color histograms onto the three color components $k, k=R, G, B$. We denote by $\left(H^{k}\left[\mathbf{I}_{\text {que }}\right], H^{k}\left[\mathbf{I}_{\text {tar }}\right]\right), k=R, G, B$, the pair of 1 D histograms which is the result of the projection $\psi^{k}$ of the pair of color histograms ( $\left.\mathbf{H}\left[\mathbf{I}_{\text {que }}\right], \mathbf{H}\left[\mathbf{I}_{\text {tar }}\right]\right)$ onto the color component $k$ :

$$
\left(H^{k}\left[\mathbf{I}_{\text {que }}\right], H^{k}\left[\mathbf{I}_{\mathrm{tar}}\right]\right)=\psi^{k}\left(\mathbf{H}\left[\mathbf{I}_{\text {que }}\right], \mathbf{H}\left[\mathbf{I}_{\mathrm{tar}}\right]\right), \quad k=R, G, B .
$$

The function $\mathbf{T}_{\text {que,tar }}$ is decomposed into three functions $T_{\text {que,tar, }}^{k}, k=R, G, B$, each of them defining an elementary transformation of the projection of the pair of color histograms onto each color component $k$. To be more specific, for determining the pair of adapted color histograms $\left(\mathbf{H}_{\text {que,tar }}\left[\mathbf{I}_{\text {que }}\right], \mathbf{H}_{\text {que,tar }}\left[\mathbf{I}_{\text {tar }}\right]\right)$, we successively apply three functions $T_{\text {que,tar }}^{k}, k=R, G, B$, to the pair of color histograms $\left(\mathbf{H}\left[\mathbf{I}_{\text {que }}\right], \mathbf{H}\left[\mathbf{I}_{\text {tar }}\right]\right)$ :

$$
\begin{aligned}
& \left(\mathbf{H}_{\text {que }, \text { tar }}\left[\mathbf{I}_{\text {que }}\right], \mathbf{H}_{\text {que,tar }}\left[\mathbf{I}_{\text {tar }}\right]\right) \\
& \quad=T_{\text {que,tar }}^{R} \circ T_{\text {que,tar }}^{G} \circ T_{\text {que,tar }}^{B}\left(\mathbf{H}\left[\mathbf{I}_{\text {que }}\right], \mathbf{H}\left[\mathbf{I}_{\text {tar }}\right]\right) .
\end{aligned}
$$

We denote by $\left(H_{\text {que,tar }}^{k}\left[\mathbf{I}_{\text {que }}\right], H_{\text {que,tar }}^{k}\left[\mathbf{I}_{\text {tar }}\right]\right)$ the pair of adapted $1 \mathrm{D}$ histograms which is the result of the projection $\psi^{k}$ of the pair of adapted color histograms $\left(\mathbf{H}_{\text {que,tar }}\left[\mathbf{I}_{\text {que }}\right]\right.$, $\mathbf{H}_{\text {que,tar }}\left[\mathbf{I}_{\text {tar }}\right]$ ) onto the color component $k$. The adapted 1D histograms contain $N$ or less nonempty bins. The parameter $N$, which is adjusted by the analyst, is lower than or equal to the number $L$ of levels. As each pair of 1D histograms $\left(H^{k}\left[\mathbf{I}_{\text {que }}\right], H^{k}\left[\mathbf{I}_{\mathrm{tar}}\right]\right)$ is transformed by a specific function $T_{\text {que,tar }}^{k}$, the determination of the pair of adapted color histograms $\left(\mathbf{H}_{\text {que,tar }}\left[\mathbf{I}_{\text {que }}\right], \mathbf{H}_{\text {que,tar }}\left[\mathbf{I}_{\text {tar }}\right]\right)$ requires the processing of three pairs of adapted 1D histograms $\left(H_{\text {que,tar }}^{k}\left[\mathbf{I}_{\text {que }}\right], H_{\text {que,tar }}^{k}\left[\mathbf{I}_{\text {tar }}\right]\right)$. Each pair of adapted 1D histograms $\left(H_{\text {que,tar }}^{k}\left[\mathbf{I}_{\text {que }}\right], H_{\text {que,tar }}^{k}\left[\mathbf{I}_{\text {tar }}\right]\right)$ is determined from the pair of 1D histograms $\left(H^{k}\left[\mathbf{I}_{\text {que }}\right], H^{k}\left[\mathbf{I}_{\text {tar }}\right]\right)$ thanks to the look- up tables $\left(\operatorname{LUT}_{\text {que,tar }}^{k}\left[\mathbf{I}_{\text {que }}\right], \mathrm{LUT}_{\text {que,tar }}^{k}\left[\mathbf{I}_{\text {tar }}\right]\right)$ :

$$
\begin{aligned}
& H_{\text {que,tar }}^{k}\left[\mathbf{I}_{\text {que }}\right](n) \\
& \quad=\sum_{\operatorname{LUT}_{\text {que,tar }}^{k}\left[\mathbf{I}_{\text {que }}\right](l)=n} H^{k}\left[\mathbf{I}_{\text {que }}\right](l), \quad n=0, \ldots,(N-1), \\
& \quad H_{\text {que,tar }}^{k}\left[\mathbf{I}_{\text {tar }}\right](n) \\
& \quad=\sum_{\operatorname{LUT}_{\text {que tar }}^{k}\left[\mathbf{I}_{\text {tar }}\right](l)=n} H^{k}\left[\mathbf{I}_{\text {tar }}\right](l), \quad n=0, \ldots,(N-1) .
\end{aligned}
$$

Equations (11) and (12) show that the adjacent bins $H^{k}\left[\mathbf{I}_{\text {que }}\right](l)\left(H^{k}\left[\mathbf{I}_{\text {tar }}\right](l)\right.$, resp.) for which the output of $\operatorname{LUT}_{\text {que,tar }}^{k}\left[\mathbf{I}_{\text {que }}\right](l)\left(\operatorname{LUT}_{\text {que,tar }}^{k}\left[\mathbf{I}_{\text {tar }}\right](l)\right.$, resp.) is equal to $n$, are merged into the bin $H_{\text {que,tar }}^{k}\left[\mathbf{I}_{\text {que }}\right](n)\left(H_{\text {que,tar }}^{k}\left[\mathbf{I}_{\text {tar }}\right](n)\right.$, resp. $)$.

\subsection{Pair of 1D look-up tables}

To determine each pair of adapted 1D histograms, $(N+$ 1) pairs of levels, denoted by $\left(X_{\text {que }}^{k}(n), X_{\text {tar }}^{k}(n)\right)$ with $n=$ $0, \ldots, N$, are selected among the $L^{2}$ possible pairs. The pair of 1D look-up tables (LUT que,tar $\left[\mathbf{I}_{\text {que }}\right], \mathrm{LUT}_{\text {que,tar }}^{k}\left[\mathbf{I}_{\text {tar }}\right]$ ) is expressed from these selected pairs of levels as

$$
\begin{aligned}
& \mathrm{LUT}_{\text {que,tar }}^{k}\left[\mathbf{I}_{\text {que }}\right](l)=n \\
& \quad \text { for } x \in\left[X_{\text {que }}^{k}(n), X_{\text {que }}^{k}(n+1)[, n=0, \ldots,(N-1),\right. \\
& \operatorname{LUT}_{\text {que,tar }}^{k}\left[\mathbf{I}_{\mathrm{tar}}\right](l)=n \\
& \quad \text { for } x \in\left[X_{\mathrm{tar}}^{k}(n), X_{\mathrm{tar}}^{k}(n+1)[, n=0, \ldots,(N-1) .\right.
\end{aligned}
$$

The selected pairs of extreme levels $\left(X_{\text {que }}^{k}(0), X_{\text {tar }}^{k}(0)\right)$ and $\left(X_{\text {que }}^{k}(N), X_{\text {tar }}^{k}(N)\right)$ are set to $(0,0)$ and $(L-1, L-1)$, respectively, so that the $1 \mathrm{D}$ look-up tables are defined for all the levels from 0 to $(L-1)$. Hence, we have to select the $(N-1)$ pairs of levels $\left(X_{\text {que }}^{k}(n), X_{\text {tar }}^{k}(n)\right), n=1, \ldots,(N-1)$.

\subsection{Selection of the pairs of levels}

In order to respect the closest rank measures assumption when the color images are similar, we propose to select these pairs of levels among those whose rank measures are the closest within the pair of color component images.

Furthermore, these pairs of levels have to be selected in order to reduce the effects of illumination changes on the adapted color histograms. These effects are effectively reduced if the intersection between the adapted color histograms reaches a higher value when the images are similar than when they are different. However, we do not a priori know if the query and target images are similar. Hence, the $3 \times(N-1)$ pairs of levels $\left(X_{\text {que }}^{k}(n), X_{\text {tar }}^{k}(n)\right), k=R, G, B$, are selected so that the three intersections between the adapted 1D histograms $H_{\text {que,tar }}^{k}\left[\mathbf{I}_{\text {que }}\right]$ and $H_{\text {que,tar }}^{k}\left[\mathbf{I}_{\text {tar }}\right]$ are high for similar or different images.

When the $3 \times(N-1)$ pairs of levels $\left(X_{\text {que }}^{k}(n), X_{\text {tar }}^{k}(n)\right)$ have been selected, we determine the 3 pairs of $1 D$ look-up tables $\left(\mathrm{LUT}_{\text {que,tar }}^{k}\left[\mathbf{I}_{\text {que }}\right], \mathrm{LUT}_{\text {que,tar }}^{k}\left[\mathbf{I}_{\text {tar }}\right]\right)$ according to (13) and (14). 
These pairs of 1D look-up tables are then used to transform the pair of color histograms $\left(\mathbf{H}\left[\mathbf{I}_{\text {que }}\right], \mathbf{H}\left[\mathbf{I}_{\text {tar }}\right]\right)$ into the pair of adapted color histograms $\left(\mathbf{H}_{\text {que,tar }}\left[\mathbf{I}_{\text {que }}\right], \mathbf{H}_{\text {que,tar }}\left[\mathbf{I}_{\text {tar }}\right]\right)$ thanks to (11) and (12).

The selection of the $(N-1)$ pairs of levels is divided into two successive steps. In order to respect the closest rank measures assumption, the first step determines the pairs of levels whose rank measures are the closest within the pair of color component images. The second step selects among these sodetermined pairs of levels those which allow to reach a high intersection between the adapted 1D histograms, in order to reduce the effects of illumination changes on the pair of adapted color histograms.

\subsubsection{Pairs of levels whose rank measures are the closest}

The first step determines the $n b_{-}$pairs ${ }^{k}$ pairs of levels whose rank measures within the pair of color component images $\left(I_{\text {que }}^{k}, I_{\text {tar }}^{k}\right)$ are the closest. We denote by $M^{k}[\mathbf{I}](l)$ the rank measure of the level $l$ within the color component image $I^{k}$, which is expressed as

$$
M^{k}[\mathbf{I}](l)=\frac{\sum_{y=0}^{l} H^{k}[\mathbf{I}](y)}{\sum_{y=0}^{L-1} H^{k}[\mathbf{I}](y)}, \quad l=0, \ldots,(L-1) .
$$

These $n b_{-}$pairs ${ }^{k}$ pairs of levels, denoted by $\left(x_{\text {que }}^{k}(a), x_{\text {tar }}^{k}(a)\right)$ with $a=1, \ldots, n b_{-}$pairs ${ }^{k}$, are those which respect the two following properties:

(i) the rank measure of $x_{\text {que }}^{k}(a)$ within the color component image $I_{\text {que }}^{k}$ is, among the rank measures of all the levels in $I_{\text {que }}^{k}$, that which is the closest to the rank measure of $x_{\mathrm{tar}}^{k}(a)$ within $I_{\mathrm{tar}}^{k}$ :

$$
x_{\text {que }}^{k}(a)=y
$$

so that

$$
\begin{aligned}
& \left\|M^{k}\left[\mathbf{I}_{\mathrm{tar}}\right]\left(x_{\mathrm{tar}}^{k}(a)\right)-M^{k}\left[\mathbf{I}_{\text {que }}\right](y)\right\| \\
& \quad=\min _{w=0, \ldots, L-1}\left\|M^{k}\left[\mathbf{I}_{\mathrm{tar}}\right]\left(x_{\mathrm{tar}}^{k}(a)\right)-M^{k}\left[\mathbf{I}_{\text {que }}\right](w)\right\|,
\end{aligned}
$$

(ii) the rank measure of $x_{\mathrm{tar}}^{k}(a)$ within the color component image $I_{\mathrm{tar}}^{k}$ is, among the rank measures of all the levels in $I_{\text {tar }}^{k}$, that which is the closest to the rank measure of $x_{\text {que }}^{k}(a)$ within $I_{\text {que }}^{k}$ :

$$
x_{\mathrm{tar}}^{k}(a)=y
$$

so that

$$
\begin{aligned}
& \left\|M^{k}\left[\mathbf{I}_{\text {que }}\right]\left(x_{\text {que }}^{k}(a)\right)-M^{k}\left[\mathbf{I}_{\text {tar }}\right](y)\right\| \\
& \quad=\min _{w=0, \ldots, L-1}\left\|M^{k}\left[\mathbf{I}_{\text {que }}\right]\left(x_{\text {que }}^{k}(a)\right)-M^{k}\left[\mathbf{I}_{\text {tar }}\right](w)\right\| .
\end{aligned}
$$

The closest rank measures assumption considers that the pixels characterized by the level $x_{\text {que }}^{k}(a)$ in $I_{\text {que }}^{k}$ and the pixels characterized by the level $x_{\mathrm{tar}}^{k}(a)$ in $I_{\mathrm{tar}}^{k}$ constitute a pair of corresponding pixels when the images are similar. Thus, the pairs of levels $\left(x_{\text {que }}^{k}(a), x_{\text {tar }}^{k}(a)\right), a=1, \ldots, n b_{-}$pairs $^{k}$, characterize pairs of corresponding pixels when the images are similar. We notice that all the levels in each color component image do not necessarily belong to a pair of levels whose rank measures are the closest. Since the number $n b_{-}$pairs ${ }^{k}$ is probably higher than the number $(N-1)$, we propose to select $(N-1)$ pairs among the $n b_{-}$pairs ${ }^{k}$ determined pairs of levels. Otherwise, all the pairs are selected and the adapted 1D histograms $H_{\text {que,tar }}^{k}\left[\mathbf{I}_{\text {que }}\right]$ and $H_{\text {que,tar }}^{k}\left[\mathbf{I}_{\text {tar }}\right]$ only contain $n b_{-}$pairs $^{k}$ bins.

\subsubsection{Selection of the $(N-1)$ pairs $\left(X_{\text {que }}^{k}(n), X_{\mathrm{tar}}^{k}(n)\right)$}

In the second step, the $(N-1)$ pairs of levels $\left(X_{\text {que }}^{k}(n), X_{\text {tar }}^{k}(n)\right)$ are selected so that the intersection between the adapted 1D histograms is as high as possible. A solution would be to select the $(N-1)$ pairs characterized by the closest rank measures among the $n b_{-}$pairs ${ }^{k}$ determined pairs of levels. This solution would not be sufficient in terms of image discrimination because it tends to select pairs of levels which represent low populations of pixels in the pair of color component images.

To cope with this problem, the proposed scheme determines the number of pixels characterized by levels of $I_{\text {que }}^{k}$ ranging from $x_{\text {que }}^{k}(a)$ to $\left(x_{\text {que }}^{k}(a+1)-1\right)$. Indeed, the intersection between the adapted 1D histograms takes into account the minimum between the number of pixels whose levels range from $x_{\text {que }}^{k}(a)$ to $\left(x_{\text {que }}^{k}(a+1)-1\right)$ in $I_{\text {que }}^{k}$ and the number of pixels whose levels range from $x_{\mathrm{tar}}^{k}(a)$ to $\left(x_{\operatorname{tar}}^{k}(a+1)-1\right)$ in $I_{\operatorname{tar}}^{k}$. In order to maximize the intersection between the adapted $1 \mathrm{D}$ histograms, the $(N-1)$ selected levels $x_{\text {que }}^{k}(a)$ are those characterized by the highest numbers of pixels. With each so-selected level $x_{\text {que }}^{k}(a)$, we associate the level $x_{\mathrm{tar}}^{k}(a)$ to constitute one of the $(N-1)$ pairs of levels $\left(X_{\text {que }}^{k}(n), X_{\text {tar }}^{k}(n)\right)$.

\section{EXPERIMENTAL RESULTS}

\subsection{Object recognition across illumination changes with the SFU database}

We propose to demonstrate the improvement of the intersection between the pairs of adapted color histograms for object recognition purpose across illumination changes. We use the Simon Fraser University (SFU) database [7] available at http://www.cs.sfu.ca/ colour/data. Its 187 images contain 17 objects lit by one of 11 available illumination sources and acquired with the same viewing conditions by one camera (see Figure 2).

For object searching, the images acquired under one illumination, called the target illumination, are considered as being the target images and one of those acquired under one of the 10 other illumination sources, called the query illumination, is considered as being the query image. So, there are $11 \times 10$ different pairs of query-target illuminations. The image retrieval is repeated for each of the 17 objects. Finally, 1870 retrievals are achieved $(17$ objects $\times 11 \times 10$ pairs of different illuminations). 


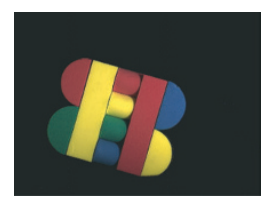

(a)

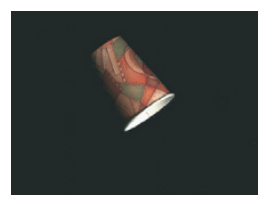

(g)

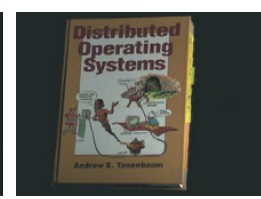

(b)

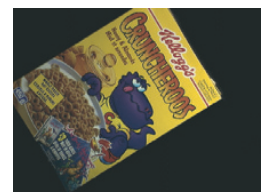

(h)

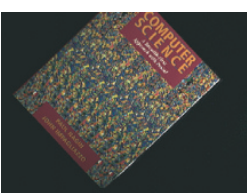

(c)

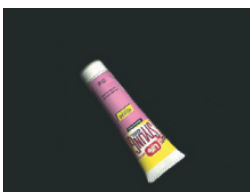

(i)

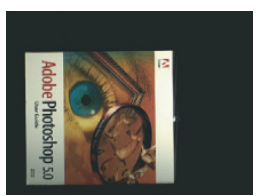

(d)

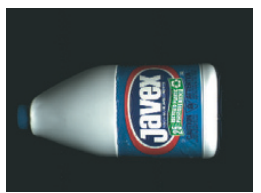

(j)

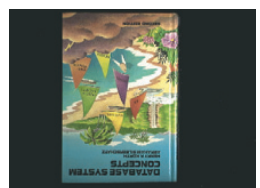

(e)

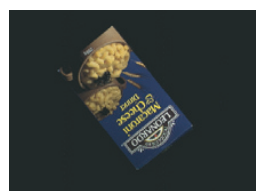

(k)

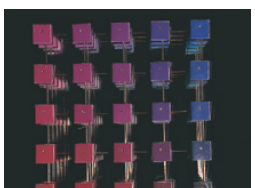

(f)

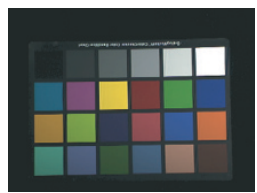

(1)

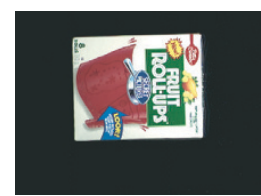

(m)

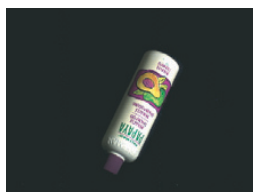

(n)

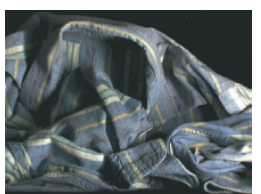

(o)

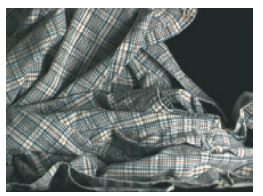

(p)

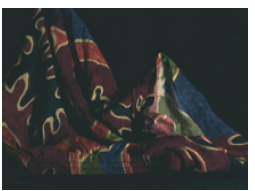

(q)

Figure 2: The 17 objects of the SFU database.

TABLE 3: Object recognition results obtained by the intersections between different histograms with the SFU database.

\begin{tabular}{lccc}
\hline Intersection between & $(N=16)$ & $(N=64)$ & $(N=256)$ \\
\hline Invariant color histograms (greyworld) & 93.85 & 80.43 & 39.73 \\
Invariant color histograms (equalization) & 89.89 & 75.08 & 48.72 \\
Adapted color histograms & 95.19 & 85.40 & 79.57 \\
\hline
\end{tabular}

For each image retrieval, the 17 target images are ordered with respect to the intersections between both their invariant and adapted color histograms and both the invariant and adapted color histogram of the considered query image. When the first ordered target image is similar to the query image, the research result is considered as perfect.

Finlayson demonstrates that the object recognition results obtained by the intersection between the invariant color histograms processed by the greyworld normalization outperforms those obtained by the intersection between classical invariant color histograms [7]. Furthermore, Finlayson concludes that the intersection between the invariant color histograms resulting from $1 \mathrm{D}$ histogram equalization provides better results than those obtained by greyworld normalization [18]. Hence, we propose to compare the results obtained by these two schemes with those obtained by the intersection between the adapted color histograms.

In Table 3 each column indicates the percentage of successful image retrievals.

Table 3 shows that, for object recognition across illumination changes, the intersection between the adapted color histograms provides better results than those obtained by the intersection between invariant color histograms, for significantly different values of $N$. Furthermore, Table 3 shows that the quality of object recognition by the intersection between the invariant color histograms is very sensitive to $N$. On the other hand, the results obtained by the intersection between the adapted color histograms remain stable when $N$ varies.

\subsection{Discussion}

The improvements provided by our scheme with this database can be explained by three main points. First, the pair of adapted color histograms determination is derived from our original model of illumination changes, which requires less restrictive assumptions than those used by the classical models. As the results obtained with the adapted color histograms are better than those obtained with the invariant color histograms resulting from 1D histogram equalization, we conclude that the possible rank measure modifications which may occur between two similar color component images, have to be taken into account by the model. Secondly, the performance reached by object recognition schemes depends on the number $(N)^{3}$ of bins of the invariant or adapted color histograms. For processing the invariant color histograms, the reduction of the number of bins from $(L)^{3}$ to $(N)^{3}$ is performed by means of a uniform quantization of the color component levels. This quantization merges adjacent bins without taking into account the color distribution. On the contrary, the selection of pairs of levels for determining the pairs of adapted color histograms 
takes into account the color distribution. Hence, the pairs of adapted color histograms do not depend on any uniform quantization step which degrades the quality of the color distribution representation. Finally, the proposed strategy based on the independent analysis of each pair of color images allows better results to be obtained than those achieved by the separate analysis of each image of the database.

\section{CONCLUSION}

In this paper, we have proposed an original approach to cope with the problem of the object recognition across illumination changes. We propose to consider each pair constituted by the query image and one of the target images instead of determining invariant color histograms for each image of the database. For each pair, the proposed scheme determines the pair of adapted color histograms whose intersection is higher when the images are similar than when they are different. This scheme is based on an original assumption which stipulates that the rank measures of corresponding pixels are the closest within similar color component images.

Although the experimental results obtained by the intersection between the adapted color histograms are satisfying, the object recognition results may be improved by adapting the pairs of color co-occurrence matrices which take into account the spatial interactions between the pixels [19].

The proposed scheme is designed for object recognition when the images contain one single object. Presently, we work on the generalization of the adapted color histograms in order to recognize several objects lit by uncontrolled illumination contained in the same image.

\section{REFERENCES}

[1] S. Tabbone and L. Wendling, "Color and grey level object retrieval using a 3D representation of force histogram," Image and Vision Computing, vol. 21, no. 6, pp. 483-495, 2003.

[2] B. Manjunath and W. Ma, "Texture features for browsing and retrieval of image data," IEEE Trans. Pattern Anal. Machine Intell., vol. 18, no. 8, pp. 837-842, 1996.

[3] D. Muselet, C. Motamed, L. Macaire, and J.-G. Postaire, "Cooccurrence matrices of color feature vectors for multi-camera vehicle identification," in Proc. Conference on Advanced Concepts for Intelligent Vision Systems (ACIVS '03), pp. 22-29, Ghent, Belgium, September 2003.

[4] M. J. Swain and D. H. Ballard, "Color indexing," International Journal of Computer Vision, vol. 7, no. 1, pp. 11-32, 1991.

[5] B. Schiele, Object recognition using multidimensional receptive field histograms, Ph.D. thesis, Institut National Polytechnique de Grenoble, Grenoble, France, 1997.

[6] G. Sharma and H. Trussell, "Digital color imaging," IEEE Trans. Image Processing, vol. 6, no. 7, pp. 901-932, 1997.

[7] G. Finlayson and G. Schaefer, "Colour indexing across devices and viewing conditions," in Proc. 2nd International Workshop on Content-Based Multimedia Indexing (CBMI '01), pp. 215221, Brescia, Italy, September 2001.

[8] G. Finlayson, S. Hordley, and P. Hubel, "Color by correlation: a simple, unifying framework for color constancy," IEEE Trans. Pattern Anal. Machine Intell., vol. 23, no. 11, pp. 12091221, 2001.

[9] G. Finlayson and S. Hordley, "Colour constancy at a pixel,"
Journal of the Optical Society of America, vol. 18, no. 2, pp. 253-264, 2001

[10] G. Finlayson, "Color in perspective," IEEE Trans. Pattern Anal. Machine Intell., vol. 18, no. 10, pp. 1034-1038, 1996.

[11] G. Finlayson, S. Chatterjee, and B. Funt, "Color angle invariants for object recognition," in Proc. 3rd IS\&T/SID Color Imaging Conference, pp. 44-47, Scottsdale, Ariz, USA, November 1995.

[12] T. Gevers and A. Smeulders, "Color-based object recognition," Pattern Recognition, vol. 32, no. 3, pp. 453-464, 1999.

[13] R. Gershon, A. D. Jepson, and J. K. Tsotsos, "From [r,g,b] to surface reflectance: computing color constant descriptors in images," Perception, pp. 755-758, 1988.

[14] R. Lenz, L. Tran, and P. Meer, "Moment based normalization of color images," in Proc. IEEE 3rd Workshop on Multimedia Signal Processing, pp. 103-108, Copenhagen, Denmark, September 1999.

[15] Z. Lin, J. Wang, and K. Ma, "Using eigencolor normalization for illumination-invariant color object recognition," Pattern Recognition, vol. 35, no. 11, pp. 2629-2642, 2002.

[16] B. Funt, K. Barnard, and L. Martin, "Is machine colour constancy good enough?" in Proc. 5th European Conference on Computer Vision (ECCV'98), pp. 445-459, Freiburg, Germany, June 1998.

[17] P. Montesinos, V. Gouet, and R. Deriche, "Differential invariants for color images," in Proc. 14th International Conference on Pattern Recognition (ICPR '98), vol. 1, pp. 838-840, Brisbane, Australia, August 1998.

[18] G. Finlayson, S. Hordley, G. Schaefer, and G. Tian, "Illuminant and device invariant colour using histogram equalisation," in Proc. 11th IS\&T/SID Color Imaging Conference, pp. 205-211, Scottsdale, Ariz, USA, November 2003.

[19] D. Muselet, L. Macaire, and J.-G. Postaire, "A new approach for color person image indexing and retrieval," Machine Graphics \& Vision, vol. 11, no. 2/3, pp. 257-283, 2002.

Damien Muselet received his M.S. (Engineer) degree from the École des Mines de Douai, France, in 2001. Now, he is a Ph.D. student in automatic control and computer science at the Institute of Technology, Université des Sciences et Technologies de Lille, France. He is a member of the LAGIS Laboratory (Laboratoire d'Automatique, Génie Informatique et Signal), Université des Sciences et Technologies de Lille, France. His research interests are object recognition across illumination changes in color images.

Ludovic Macaire received the M.S. (Engineer) degree in computer science from the UTC engineering school, Compiegne, France, in 1988; the Ph.D. degree in computer science and control from the Université des Sciences et Technologies de Lille, France, in 1992, and the Habilitation à Diriger des Recherches from the Université des Sciences et Technologies de Lille, France, in 2004. Since 1993, he has been an Associate Professor at the LAGIS Laboratory (Laboratoire d'Automatique, Génie Informatique et Signal), Université des Sciences et Technologies de Lille, France. His research interests include color representation and color image analysis applied to segmentation and retrieval.
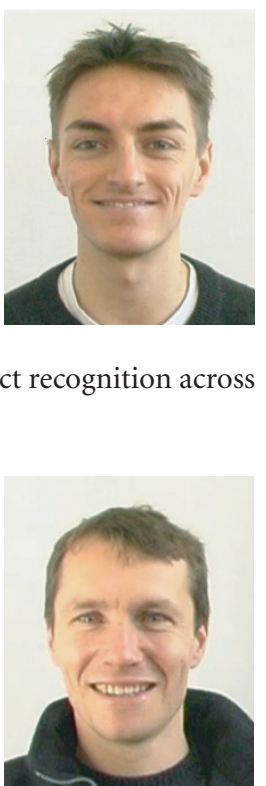
Jack-Gérard Postaire received his M.S. (Engineer) degree from the École Centrale de Lille, France, in 1970, and his Docteur es Sciences degree from the Universite des Sciences et Technologies de Lille, France, 1981. $\mathrm{He}$ is a Professor in automatic control and computer science at the Institute of Technology, Université des Sciences et Technologies de Lille, France. He has been at the Université des Sciences et Technologies de Lille,

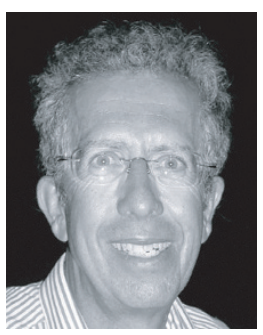

France, since 1982, when he was promoted to the rank of Professor. Prior to that, he was an Assistant Professor at the Faculty of Sciences of Rabat, Morocco, from 1976 to 1982 and an Associate Professor at the University of Sherbrooke, Canada, from 1972 to 1974. He is presently the Head of the "Vision and Image" research group at the LAGIS Laboratory (Laboratoire d'Automatique, Génie Informatique et Signal) UMR CNRS 8146, Université des Sciences et Technologies de Lille, France. The main topics addressed by the group are color image analysis, dynamic scene analysis, real-time image processing for quality control in industrial processes and security in transportation systems, multidimensional data analysis, and pattern recognition. 